\title{
Health Care Finance and the Early Adoption of Hospital Information Systems
}

\author{
Ron Borzekowski * \\ Board of Governors of the Federal Reserve System
}

\begin{abstract}
This study examines the adoption of hospital information systems (HIS), specifically focusing on the connection between the financing of health care and the adoption of these new technologies. Using a recently uncovered dataset detailing the systems installed at over 2300 hospitals, the results indicate that state price regulations slowed the adoption of these systems during the 1970's. In contrast, hospitals increased their adoption of HIS in response to the implementation of Medicare's prospective payment system. The evidence suggests that in the early years, these systems did not have the ability to save sufficient funds to justify their expense and adopters, in particular not-for-profit hospitals, were motivated by factors other than cost. By the early 1980's, this had changed: hospitals with the greatest incentives to lower costs were now more likely to adopt these technologies.
\end{abstract}

\footnotetext{
${ }^{*}$ Board of Governors of the Federal Reserve System, Washington, DC 20551. e-mail: ron.borzekowski@frb.gov. The views expressed in this paper are those of the author and do not necessarily reflect those of the Board of Governors or the staff of the Federal Reserve System. Paul Zarins and the Stanford University Libraries were very kind and helpful in procuring the data for this project and I am very grateful. Many thanks as well to Frank Wolak for his help and to participants at the Stanford University Public Lunch and IO Workshop for the chance to present an earlier draft. All errors my own.
} 


\section{Introduction}

Hospital information systems (HIS) are computers and related technologies used in the administration and management of the hospital as well as the provision of patient care. These systems include the hardware and software used to perform purely administrative tasks such as patient billing as well as more clinical systems used to order medical tests or manage ancillary departments. By 1968, nearly $24 \%$ of U.S. hospitals had adopted some HIS technologies (Herner and Company 1970), and by 1979, the number had grown to nearly 68\% (Sheldon I. Dorenfest \& Associates, Ltd. 1985). By the end of the 1990's nearly every hospital had some HIS applications.

Even with this seemingly rapid diffusion, HIS spending by hospitals in the United States lagged that of other industries. Historically, hospitals spent roughly $2 \%$ of operating expenses on IT (Sheldon I. Dorenfest \& Associates, Ltd. 1985, Kennedy 1986), far below the $4 \%$ in the insurance industry or the $12 \%$ in banking (see full study cited in Kennedy (1986)). Still, the overall size of the industry makes the magnitude of these expenditures quite large. In 1983, HIS expenditures for hardware, software and related expenses at acute care hospitals was nearly $\$ 2$ billion (Sheldon I. Dorenfest \& Associates, Ltd. 1985). In 1996, HIS spending was over $\$ 12$ billion (Dorenfest 1997).

Given the large amounts spent on these technologies, it is important that hospitals face incentives that yield efficient adoption of HIS. In much the same way that the early financing of care led to the 'medical arms race,' or that prospective payment systems limited the adoption of high cost equipment, the financing regime is likely to have impacted the adoption of these more 'managerial' technologies.

This study examines the influence of health care finance on the adoption of hospital information systems during the 1970's and early 1980's, specifically focusing on the effect of Medicare's prospective payment system, state-level policies, and general market conditions. ${ }^{1}$ During the early 1980's, hospitals were preparing for the radical shift to reimbursement depending only on the diagnosis of the patient and independent of the actual resources used to treat him or her. The burden of lowering costs now lay upon the hospital. To do so, automated hospitals could utilize current

\footnotetext{
${ }^{1}$ Prior research has tried to uncover the determinants of HIS adoption, yet none has directly focused on the financial incentives facing hospitals. See Mahajan and Schoeman (1977), Globerman (1982) Moch and Morse (1977), and Kimberly and Evanisko (1981).
} 
IT, while the remaining hospitals had the option of investing in the automation of the hospital. At this same time, there was an arguable increase in IT expenditures by hospitals of over $20 \%$ (Dorenfest 1995) as well as a greater focus on automating non-administrative areas of the organization. The study hopes to determine whether this spike in adoption was tied to the impending change in the financing regime.

The second goal of this study is to investigate whether state-level health care finance policies and other state factors influenced HIS adoption. During the 1970's several states had introduced prospective payment systems in an effort to control costs. These programs varied widely along several dimensions: the payers covered by the legislation, the method for setting prospective rates and the degree to which the program was mandatory. Certificate of need (CON) regulations were also adopted at the state level, and again variations in the specific programs created further heterogeneity among hospitals. At the market level, both HMO penetration as well as the percentage of individuals with health insurance also varied. Together, this variation in state-level regulation and market conditions created a situation where hospitals faced differing incentives for adoption.

The HIS data for this study comes from the National Survey of Hospital Data Processing, a recently uncovered series of surveys regarding hospital information systems. Conducted annually from 1982 through 1985 by Ralph Grams at the University of Florida, the survey was sent to the approximately 7000 hospitals in the United States. In total, nearly 2300 hospitals responded to the surveys, providing data regarding the hardware and software installed in each institution. In particular, the data focuses on six specific components of these systems: finance systems, admissions/discharge/transfer (ADT) systems, order entry systems, laboratory systems, pharmacy systems and radiology systems. These components span both the administrative and clinical sections of the hospital, and at the time formed the bulk of hospital computing.

While the dataset is quite extensive, the sampling frame results in an unbalanced panel with hospitals responding to the survey a varying numbers of times. Further, only partial information about the timing of adoption is available. To resolve these difficulties and to analyze the determinants of adoption prior to the sampling period, the analysis includes both discrete and continuous hazard models, each designed to best utilize the available data. The discrete model assumes a piecewise- 
constant hazard function and is estimated using conditional maximum likelihood. It avoids any functional form assumptions and includes the measurement of time-varying effects. However, it does do not utilize all the available data. The continuous model utilizes the additional data on the timing of adoption by assuming a log-logistic hazard function.

The results provide evidence that HIS adoption was affected by the financing regime. In the 1970's, hospitals in price regulated states were slower to adopt these technologies, as were government and for-profit hospitals. In contrast, hospitals most impacted by the implementation of the Medicare prospective payment system were faster to adopt HIS. Neither the state-level controls on capital expenditures nor the other financing variables are shown to have significant associations with HIS adoption.

Taken together, the results suggest that the ability of HIS to reduce costs relative to their price increased over this period. During the 1970's, hospital adoption was motivated by concerns other than cost reduction and as a result, price regulation limited those investments. Hospitals in better financial situations seem to have been able to adopt and experiment with HIS. By the early 1980's, this had changed and hospitals with the greatest incentives to lower costs were more likely to adopt these technologies.

The rest of this study is structured as follows. Section 2 discusses the relevant financing regimes

and the incentives to adopt these systems. Related work on this topic is also addressed. Section 3 then describes the data used in this study while Section 4 details the models used for the estimation and the basic results. Section 5 discusses the results and offers some conclusions.

\section{Health Care Finance and the Adoption of Technology}

To analyze the incentives hospitals faced to adopt hospital information systems requires information in three critical areas: the financing regime, the role of information technology in hospital production and the motivations of hospitals. Each of these is discussed below: the three are then connected to form specific hypotheses which are tested in the following sections.

Throughout the 1970's, states began to increase the reporting requirements for hospitals and 
several states imposed hospital price regulation. In all cases, the goal was the reduction of then escalating hospital costs. However, these programs varied along several dimensions: the payers covered by the legislation, the method for setting prospective rates and the degree to which the program was mandatory. ${ }^{2}$ The variations imply that certain states had more stringent regulations than others.

In October 1982, the Tax Equity and Fiscal Responsibility Act (TEFRA) was passed, weakening the connection between a hospital's costs and its Medicare reimbursement. With the passage of PL 98-21 in April 1983, the full Medicare PPS system was implemented and hospitals were no longer able to recover the full cost of treating Medicare patients, but instead faced a fixed diagnosis-based reimbursement schedule. The PPS system was effective for all fiscal years beginning on or after October 1983 except for those hospitals in waiver states with strong all-payer price regulations. For these states, the changes were delayed by several years. As with the state programs, the incentive to reduce costs were greatly increased by the new PPS system. The expansion of HMO's and capitated payments during the late 1980's and 1990's only continued this trend.

State-level certificate of need regulation was also implemented in this period, requiring hospitals to get state approval for capital expenditures. As with price regulations, the state CON programs were quite varied as states set different limits for the types or amounts of expenditures subject to these regulations (Feinglass et al. 1990).

The effect of these financial incentives on the adoption of other technologies has been extensively studied. Romeo, Wagner and Lee (1984) specifically address state prospective payment programs, finding that hospitals in states with greater pressures on reimbursement are slower to adopt costincreasing technologies and faster to adopt cost-saving technologies.

More commonly, research has focused on the adoption of larger and more expensive technologies. Teplensky et al. (1995) conclude that a shift to prospective payment, as well as more stringent rate regulation by the states, lowered the adoption rates for MRI machines. Baker (2001) finds that increased managed care penetration limited MRI diffusion while a recent study by Culter and McClellan (1996) indicates the insurance variation influenced the adoption and use of angioplasty

\footnotetext{
${ }^{2}$ For comprehensive summaries of these regulations, see Rosko and Broyles (1988) and Gaumer et al. (1989).
} 
for heart attack patients.

To assess the impact that these regulatory changes would have on HIS adoption, it is important to understand the role of these systems in hospital production. In the simplest terms, these systems are best thought of as cost reducing and/or quality improving technologies. ${ }^{3}$

Hospital information systems can directly reduce costs in two basic ways: by reducing administrative costs or by solving the agency problem within the hospital. Hospitals are comprised of a multitude of specialized departments and suppliers requiring that large amounts of clinical as well as financial data be exchanged. Automating these processes may reduce costs as less paper is generated, as fewer mistakes are made and as information is transferred faster. A series of studies in the late 1960's and early 1970's claimed that up to $30 \%$ of hospital costs were dedicated to the collection, storage and retrieval of information. Others noted that up to $30 \%$ of personnel time was also dedicated to these activities (Mahajan and Schoeman 1977).

These systems may also reduce costs by helping hospital administrators solve the agency problem within the hospital. In the United States, health care is financed by third-parties, creating an environment where incentives may not align between doctors, patients, administrators and thirdparty payers. The most common of these is the chance for doctors to overuse hospital resources for which he or she bears no cost. To the extent that HIS can mitigate such moral hazard problems, the hospital will have the incentive to adopt.

Computers can also increase the quality of medical care. This was the goal of many of the pioneers in medical informatics or clinical systems development. From early on, they argued that quality improvements from hospital information systems would emerge from the improved recordkeeping and decreased mistakes engendered by more administrative systems, as well as from clinical systems designed to aid in the provision of medical care. Federal funds were devoted to this effort and academic centers for this area of research were developed beginning in the early 1960's (Collen 1995). ${ }^{4}$ Even with these efforts, however, it is unlikely that HIS at this early time would

\footnotetext{
${ }^{3}$ HIS can also increase revenue by improving the accuracy of billing. For expositional purposes, and to the extent that this revenue effect is real, it is viewed as a reduction in the 'cost' of un-billed items.

${ }^{4}$ It is interesting to note that two very recent Institute of Medicine studies again called for the use of information technology to improve the quality of care. See Kohn, Corrigan and Donaldson, eds (2001) and Committee on Quality Health Care in America, Institute of Medicine (2001).
} 
have much impact on quality. The diffusion of clinical systems was still limited, and those that were installed had more of an coordination focus. Although little research exists directly examining this question, the prevailing wisdom is that HIS were more likely to impact costs rather than quality of care in the early period.

The extent to which either the cost effect or the quality effect will motivate a given hospital to adopt HIS depends on the motivations of the firm. Unfortunately, the precise maximand for hospitals, the majority of which are not-for-profit, is far from certain and has been debated in the literature for some time. ${ }^{5}$ In this paper, all hospitals are assumed to value a weighted sum of profits and quality. Further, for-profit hospitals are assumed to place greater weight on profits than their not-for-profit counterparts. Based on the conventional wisdom described above, a last assumption is that the ability of HIS to effect hospital quality was negligible during this period.

In this framework, the adoption of HIS at a given hospital is driven by the insurance mix of its patients and the hospital's relative valuation of profits. The precise relationships among these variables is detailed in the two hypotheses that follow. The first describes adoption as a function of the prospective payment system while the second describes adoption as a function of for-profit status.

Hypothesis 1. Assuming that hospital information systems are primarily cost reducing technologies, the likelihood of adoption in any given year is increasing in the percentage of prospective payment patients.

The idea behind this hypothesis is simple. Regardless of the costs incurred to treat a fee-forservice patient, the hospital will recover those costs along with some markup. If the markup is a positive percentage of the costs, it is in the hospital's interest to maintain higher costs and thus higher profits. In contrast, under prospective payment, the hospital keeps any of the savings. This forces a tradeoff where the hospital keeps any IT-related cost reductions from PPS patients while losing the markup on all fee-for-service patients. Assuming HIS lower costs, hospitals with higher

\footnotetext{
${ }^{5}$ In his seminal work, Newhouse (1970) posited the not-for-profit hospital as maximizing some combination of profits and quantity subject to a zero-profit constraint. Other authors such as Pauly and Redisch (Pauly and Redisch 1973) argued for income per doctor as the maximand while still others include labor or capital slack in the objective function. See Sloan (2000) for a complete discussion of this issue.
} 
percentages of PPS patients will be more likely to adopt.

The implications of this are that hospitals in PPS states are expected to have adopted earlier. As well, those hospitals with greater percentages of Medicare patients are the ones expected to adopt in the early 1980's in advance of the PPS introduction.

The comparative statics regarding the for-profit status of a hospital are not as clean and depend on the mix of patients. Assuming for-profit hospitals place greater weight on profits, the effects described above will be greater for for-profit hospitals. At the extreme where profits are the only objective, the loss of FFS revenue or the cost savings from PPS will be all that matter. Thus, in an environment with only FFS reimbursement, for-profit hospitals should be slower adopters. The opposite result should hold in an environment with only prospective reimbursement. More formally,

Hypothesis 2. Assuming that HIS are primarily cost reducing technologies, the difference in incentives to adopt HIS between for-profit and not-for-profit hospitals depends on the patient mix. In environments with a high percentage of fee-for-service patients, for-profit hospitals are less likely to adopt a given system than not-for-profit hospitals. Conversely, in environments with a high percentage of PPS patients, for-profit hospitals will be more likely to adopt than their not-for-profit counterparts. ${ }^{6}$

While this hypothesis is stated as a cross-sectional distinction, it should also hold dynamically: as time goes on and a greater percentage of any hospital's population is likely to be covered by PPS, for-profit hospitals are expected to shift from being less likely to adopt to being more likely to adopt. ${ }^{7}$

\footnotetext{
${ }^{6}$ This hypothesis conjectures that behaviors vary between for-profit hospitals and not-for-profit hospitals. While many theories yield this result, empirical work to date often fails to find a difference. See Sloan (2000) for thorough review of this issue.

${ }^{7}$ Neither of these hypotheses is likely to be controversial. However, it is important to note two key assumptions in the analysis: 1) that HIS do not directly influence the hospital's demand and 2) that hospitals do not face financing constraints. To the extent that borrowing constraints exist, requiring the financing of IT from retained earnings, the effects of the financing regime may be different.
} 


\section{Data}

This section describes the data used in this study. Since the HIS data is less familiar to most readers, it is discussed first. In particular, the precise construction of the two different dependent variables is described. The independent variables are described at the end of this section.

\subsection{Hospital Information System Data}

The HIS dataset comes from the National Survey on Hospital Data Processing. This survey was conducted annually by Ralph Grams at the University of Florida from 1982-1985, and published by Medical Systems Research. In each year, the team surveyed all 7000 hospitals in the United States and received between 1200 and 1500 responses. Respondents provided data on the hardware and software in place in several functional areas including financial systems, ADT systems, order entry systems, laboratory systems, radiology systems and pharmacy systems. Complete descriptions of the questionnaires as well as summaries of the results can be found in Grams et al. (1985) and Grams and Peck (1986).

Because of data limitations, only the first three years of the survey (1982-1984) are utilized in the current study. The sample is further restricted to short-term general non-federal hospitals with at least 100 beds. This last restriction is driven by the research showing that larger hospitals

behave very differently than smaller ones (e.g. see Culter and McClellan (1996) and Carey and Stefos (1992)).

The final sample consists of 1452 hospitals, roughly $48 \%$ of the 3007 short-term general nonfederal hospitals with at least 100 beds. Table 2 provides a comparison of the sample to the universe of similar AHA hospitals. On average, hospitals in this sample are slightly larger and more likely to be not-for-profit enterprises. This is expected since not-for-profit institutions are less likely to view disclosure of this information as competitively disadvantageous. 


\subsection{Adoption Data}

In this study, the dependent variable is the time of adoption for the six hospital information systems. The features of this dataset, however, do not allow 'perfect' measures of these variables. Instead, two separate measures are created, one based solely on the presence or absence of the system at the time(s) the hospital was sampled and one based on the date of adoption detailed in the dataset. The precise issues involved with each of these are the subject of this section and are discussed again in the Section 4.

Because the survey was sent to all hospitals in each year, any given hospital may appear in the dataset in any or all of the three years. As an example, 312 hospitals returned the survey only in 1982 while 255 appear in the sample in all three years. The complete distribution is detailed in Table 1.

Table 1: Frequency of Hospital Appearance in the Annual Surveys and Classification of Adoption Times

\begin{tabular}{|c|c|c|c|c|c|}
\hline \multirow{2}{*}{$\begin{array}{c}\text { Sampling } \\
\text { Type }\end{array}$} & \multicolumn{3}{|c|}{ Years in Survey } & \multirow{2}{*}{$\begin{array}{l}\text { Frequency } \\
\text { in Sample }\end{array}$} & \multirow{2}{*}{$\begin{array}{l}\text { Adoption } \\
\text { Times }\end{array}$} \\
\hline & 1982 & 1983 & 1984 & & \\
\hline 1 & $\mathrm{X}$ & & & 312 & $\mathrm{~B} 81, \mathrm{~A} 81$ \\
\hline 2 & & $\mathrm{X}$ & & 226 & B82, A82 \\
\hline 3 & & & $\mathrm{X}$ & 196 & B83, A83 \\
\hline 4 & $\mathrm{X}$ & $\mathrm{X}$ & & 220 & B81, I82, A82 \\
\hline 5 & $\mathrm{X}$ & & $\mathrm{X}$ & 90 & $\mathrm{~B} 81, \mathrm{I} 82^{*}, \mathrm{I} 83^{*}, \mathrm{~A} 83$ \\
\hline 6 & & $\mathrm{X}$ & $\mathrm{X}$ & 153 & $\mathrm{~B} 82, \mathrm{I} 83, \mathrm{~A} 83$ \\
\hline 7 & $\mathrm{X}$ & $\mathrm{X}$ & $\mathrm{X}$ & 255 & B81, I82, I83, A83 \\
\hline
\end{tabular}

If a hospital is sampled in a given year, one can observe whether or not they have installed a given system as of that date. However, the heterogeneity in the first and last time a hospital is seen leads to varying information regarding the timing of adoption. For example, the 312 hospitals that answered only the 1982 survey can be divided into those that adopted during or before 1981 (B81) and those that adopted after 1981 (A81). In contrast, the hospitals that returned all three surveys can be determined to have adopted during or before 1981 (B81), in 1982 (I82), in 1983 (I83), or after 1983 (A83). (Each of these classes of hospitals is referred to as a sampling type.) In the discrete hazard model below, this information forms the basis of the dependent variable: the presence or absence of the system at the time of the sample is used to determine if the hospital 
adopted before or during 1981, in 1982 , in 1983 , or after $1983 .^{8}$

The second dependent variable is derived from the adoption date for the current system as reported by the hospital. While these dates offer more information with which to analyze the determinants of IT adoption, they are imperfect measures in two critical ways. First, they are present for only a subset of the data. More importantly, the date is the adoption date of the current hardware and/or software configuration. It is possible, and even likely for the more diffused systems, that a hospital has upgraded in the past and that the specified date of adoption is not the first time the application was automated. In essence, the date given is an upper bound on the true adoption time for the given system.

The continuous model uses these adoption dates, and for dates preceding the first time a hospital is surveyed, treats them as left censored. For example, a hospital that first returns a survey in 1983 may indicate that it adopted a laboratory system in 1980. This date is assumed to be the latest possible date of adoption as the hospital may first have automated in 1974 and then upgraded to the current system in 1980. Adoption dates that are after the first survey date are treated as true adoption dates rather than as censored values. Note that to the extent that the left censored values are the true first adoption dates, this method will overstate the rate of IT diffusion. Nevertheless, these dates do provide more detailed information regarding the timing of adoption than the first set of dependent variables described above.

In summary, none of the data allows a precise measure of adoption for these systems. The variables based upon the presence/absence of the system use limited information while the variables based upon the adoption date may overstate the true timing of adoption. For robustness and to gain the best insight into HIS adoption, this study uses both of these sets of data. Section 4 details the precise way this is done.

\subsection{Independent Variable Data}

The independent variables in these regressions are divided into three sets and have been compiled from several sources. The first set is designed to capture the regulatory environment surrounding

\footnotetext{
${ }^{8}$ Adoption refers to the first installation of the given system. No distinction is made between in-house computers and the contracting of service bureaus for the same functions.
} 
the hospital. The second set captures other features of health care finance that may also impact hospitals technology choice while the third set includes several control variables. Each of these is discussed in turn.

\subsubsection{Regulatory Variables}

The regulatory variables capture the effects of state price regulation, certificate of need regulation and the cost pressures imposed by the adoption of Medicare's PPS. ${ }^{9}$

To capture the effects of state rate regulations, states are placed into three groups, depending on the stringency of their regulations. ${ }^{10}$ The first group, PPS_ST1, includes hospitals in one of the eight states that had mandatory price regulations as of 1980. These include Connecticut, Maryland, Massachusetts, New Jersey, New York, Rhode Island, Washington and Wisconsin. It is anticipated that hospitals in these states faced greater incentives to cut costs and may have started on the automation path sooner. The second group, PPS_ST2 includes hospitals in one of the other eight states which had either mandatory hospital rate review programs or an extensive partial payer system. These include Arizona, Indiana, Kentucky, Minnesota, Nebraska, Oregon and Virginia. Colorado is also included in this second group; while its program was more similar to those in the first group of states, it had terminated that program as of 1980.

To capture the effect of CON regulations, states are also grouped by the stringency of their regulations. To create this measure, Feinglass et al. (1990) assigned each state into one of four categories for each of five factors: capital expenditure thresholds, medical equipment thresholds, new institutional service thresholds, percentage of hospital applications approved and state CON budget per non-federal hospital. By assigning zero to three points for each group, these authors created a scale rating each state from zero to fifteen; the higher numbers imply a higher stringency of regulation.

The current study uses these values broken down into three groups: 0-5 (CONO), 6-10 (CON1)

\footnotetext{
${ }^{9}$ While the hypotheses generated earlier specify precise relationships between adoption and the percentage of patients under PPS, the regressors used here are based on a broader notion of stringency that incorporates not only the patient base, but also the level of reimbursement. The broader view is theoretically correct in a dynamic setting, although such a theory is more complex than the one described earlier.

${ }^{10}$ This specification is derived from that in Feinglass et al. (1990) although the grouping of states is different due to the different time period.
} 
and 11-15 (CON2). The groupings are meant to provide a parsimonious set of variables that captures the heterogeneity across hospitals, maintains the ordering among the state regulations, and avoids relying on the cardinality of the index. ${ }^{11}$

To be valid regressors, these indicator variables must be exogenous. The omission of any variable that jointly determines HIS adoption as well as state regulations would lead to correlation with the error term and inconsistent results. States with rapidly rising costs, for example, may be the ones to adopt price regulations. Hospitals in these states may also be the ones to adopt HIS, not in response to the regulations, but rather in response to the other factors driving the price increases. It is therefore important to understand the demand for these regulations.

Unfortunately, the research on the determinants of state-level regulation is quite limited. Salkever (2000) recently summarizes this literature noting that 'the desire to constrain Medicaid spending was the key factor in promoting state legislative adoption of regulatory controls, but that indicators of general political preferences also had significant explanatory power.' Neither of these factors is likely to have directly impacted IT adoption. The level of Medicaid spending is primarily determined by the income level of the state. Unless the care of the poor is differentially impacted by HIS, this should not have any direct impact (aside from the reimbursement scheme captured by the state variables). Similarly, no direct connection seems to exist between the political leanings of the state and the costs or benefits of HIS adoption.

The last relevant regulatory issue during this period is the potential cost impact created by the Medicare PPS. To capture the degree to which this differentially effected hospitals, we create a Medicare 'bite' variable MBITE, fashioned after the fiscal pressure index calculated by Hadley, Zuckerman and Feder (1989). This measure is defined as the anticipated loss in reimbursement from Medicare expressed as a percentage of the hospitals annual expenses. Specifically, we define:

$$
\operatorname{MBITE}_{j}=\frac{\left(C P C A S E_{j}-P P C A S E_{j}\right) *\left(M C A D M 82_{j}\right)}{E X P T O T_{j}}
$$

\footnotetext{
${ }^{11}$ See Borzekowski (2002) for a listing of the actual values for each of the state-level regulation indicator variables.
} 
The average cost per case CPCASE, as well as the average PPS reimbursement per case $\mathrm{PPPCASE}_{j}$, are estimated using data from the 1982 American Hospital Association (AHA) Annual Survey of Hospitals and data from the Health Care Financing Administration (HCFA). The difference between these two values is an estimate of the lost reimbursement from the switch to PPS for a hospital's average Medicare patient. Before PPS, the hospital would be reimbursed costs. After the change, the reimbursement is fixed based only upon the diagnosis. This change is captured in the difference of these two terms, which is then multiplied by the expected number of Medicare patients, $M C A D M 82$ to estimate the total change in revenue at the hospital. To normalize this value it is divided by EXPTOT, the total operating expenses at the hospital. A more detailed explanation of these calculations is included in Borzekowski (2002).

As Table 2 indicates, the average MBITE value is approximately -.043 indicating a loss of $4.3 \%$ of expenses. However, it ranges widely. In order to avoid relying on the cardinality of this figure, $M B I T E$ is discretized into five dummy variables. $M B O$ is defined as 1 for all hospitals where MBITE was positive i.e. those with below average costs who stood to gain from the onset of PPS. $M B 1$ includes those hospitals in waiver states who by definition have MBITE equal to 0 . The remaining three groups have potential revenue impacts of between $0 \%$ and $8 \%$ (MB2), $8 \%$ and $14 \%$ (MB3), and over 14\% (MB4). While this grouping may introduce some measurement error, it allows for a non-linear relationship between MBITE and the probability of adoption, preserves the rough ordering of hospitals, and offers a simple way to capture these effects. ${ }^{12}$

\subsubsection{Finance Variables}

As additional measures of heterogeneity in the fiscal environment facing hospitals, this study uses the percentage of the state population enrolled in health maintenance organizations as well as the percentage of the local population with health insurance. The former variable, $H M O_{-} P E N$ is measured at the state level as of 1980 from data published by Interstudy. This is the only date within the study period for which the data is available. Assuming that patterns of HMO enrollment did not vary over the surrounding years, this measure will accurately capture variation

\footnotetext{
${ }^{12}$ Other possible approaches would be to use a polynomial in MBITE in order to create a flexible non-linear index or to use the rank order of MBITE as the regressor.
} 
Table 2: Variable Definitions and Summary Statistics

\begin{tabular}{|c|c|c|c|}
\hline Variable & Definition & $\begin{array}{l}\text { Universe } \\
(\mathrm{N}=3007)\end{array}$ & $\begin{array}{l}\text { Sample } \\
(\mathrm{N}=1452)\end{array}$ \\
\hline \multicolumn{4}{|c|}{ Control Variables } \\
\hline NFP & $=1$ if hospital is not-for-profit & .699 & .768 \\
\hline PROFIT & $=1$ if hospital is for-profit & .119 & .044 \\
\hline GOV & $=1$ if hospital is government owned & .182 & .188 \\
\hline $\mathrm{TSCH}$ & $\begin{array}{l}=1 \text { if hospital is member of Council of Teaching Hos- } \\
\text { pitals }\end{array}$ & .101 & .129 \\
\hline RURAL & $=1$ if hospital is not located in an MSA & .279 & .257 \\
\hline BDTOT & Total number of beds at the hospital & 276 & 305 \\
\hline \multicolumn{4}{|c|}{ Regulatory Variables } \\
\hline PPS_ST1 & $=1$ if hospital is a state with stricter price regulations & .197 & .223 \\
\hline PPS_ST2 & $=1$ if hospital is in state with weaker price regulations & .120 & .121 \\
\hline CON1 & $\begin{array}{l}=1 \text { if hospital is in a state with weaker CON regula- } \\
\text { tions }\end{array}$ & .456 & .488 \\
\hline CON2 & $\begin{array}{l}=1 \text { if hospital is in a state with stricter } \mathrm{CON} \text { regula- } \\
\text { tions }\end{array}$ & .320 & .349 \\
\hline MBITE1 & see text & -0.036 & -0.043 \\
\hline \multicolumn{4}{|c|}{ Finance Variables } \\
\hline HMO_PEN & Percentage of pateints in the state covered by an HMO & 0.036 & 0.031 \\
\hline $\mathrm{COV}$ & $\begin{array}{l}\text { Percentage of patients in the state covered by health } \\
\text { insurance }\end{array}$ & 0.852 & 0.857 \\
\hline
\end{tabular}

in the percentage of the hospitals patients for which payments are capitated.

The insured percentage, $C O V$, is calculated from the 1980 Current Population Survey (CPS). ${ }^{13}$

For hospitals in MSA's, this number is the percentage of individuals with any form of health insurance. For other hospitals, it is the percentage of insured individuals in the non-MSA portion of the state.

\subsubsection{Controls}

All of the control variables are taken from the AHA data. To test the hypotheses offered earlier, the for-profit status of the hospital, PROFIT is included as a regressor. It is expected that these hospitals adopt HIS at a slower rate than other hospitals in the earlier periods and at a faster rate

\footnotetext{
${ }^{13}$ The 1980 CPS has data as of 1979 , the earliest time that health insurance data was included on the CPS. To be consistent, the 1981 CPS should have been used, however the survey in that year was missing one insurance-related question, making the data invalid.
} 
later on.

To control for the size of the hospital, all of the models use the log of the number of beds, $L B D$. If HIS exhibit increasing returns to scale, the average cost for large users should be lower. As such, adoption should be faster in larger hospitals, a result found in most prior research. Dummy variables are also included for whether the hospital is government owned, GOV, and whether the hospital is a teaching facility, TSCH. These latter hospitals are likely to be technologically more advanced and may therefore have adopted earlier.

In other studies of hospital technology adoption, demand side variables are often included as predictors. Variables such as the number of elderly patients, or median income of patients, in the hospital's area are included under the theory that an overall shift in the demand for health care will also shift demand for these technologies. This connection is much more tenuous in the case of hospital information systems. During this time period, most hospital information systems were invisible to patients, As a result, it was unlikely to directly influence demand.

Variables for the degree of competition for doctors that each hospital faced are also omitted. For other technologies, it is reasonable to assume that doctors will be attracted by the new 'toys.' Again, with IT, this connection is less obvious. A high degree of computerization may attract doctors if it signals quality; the potential monitoring of doctors, however, may steer them away from the given hospital. These variables were included in some preliminary analyses with very limited results; therefore they are not included in the current study.

Finally, Hypothesis 2 states that in areas with a high percentage of PPS patients, for-profit hospitals should adopt faster than others. The best test for this would be an interaction term between the regulatory variables and the for-profit status of the hospital. Unfortunately, the low percentage of for-profit hospitals prohibits the use of these variables. For example, fewer than fifteen for-profit hospitals are in the PPS_ST1 states and around twenty are in any single Medicare bite group. 


\section{Analysis}

As described above, the sampling frame for this dataset allows the construction of two measures for the adoption times of these six hospital information systems. In this section, these differing measures are used in two separate estimations, designed to answer slightly different questions and to provide robustness to the analysis. The first estimation, a discrete piecewise-constant hazard model, uses the measures based upon the presence or absence of the systems. The second estimation uses the adoption dates in a continuous hazard model. Together, these results provide a complementary set of analyses to ascertain whether HIS adoption was influenced by the financing of health care. A full discussion of these results follows while their interpretation is delayed until the following section.

\subsection{Discrete Hazard Model}

The piecewise-constant hazard model uses only the presence or absence of the systems during the sample period to generate the independent variables. This eliminates any assumptions regarding the precise timing of adoption and allows the focus to be the implementation of Medicare PPS and its effect on HIS adoption. It also eliminates the need for functional form assumptions.

The basic components of the empirical model are two conditional probabilities: that of adopting before or during $1981\left(P^{1}\right)$, and that of adopting during 1982 or 1983 conditional on not having adopted before then $\left(P^{2}\right)$. Each of these probabilities is then parameterized in a probit model as:

$$
P^{i}=\Phi\left(\beta_{i}^{\prime} X\right) \quad i \in 1,2
$$

The latter probability is further separated into the probability of adopting in $1982\left(\alpha P^{2}\right)$ and that of adopting in $1983\left((1-\alpha) P^{2}\right)$. This is done to economize on parameters since the alternative would be to specify a third probability for adoption in 1983. This modeling choice should be innocuous as the factors influencing adoption in 1983 should not vary much from those in 1982.

As discussed above and detailed in Table 1, the data allow the identification of eight adoption 
times: $B 81, B 82, B 83, A 81, A 82, A 83,182$, and 183 . The possible adoption times for a given hospital depends its sampling type. Thus, for Type 1 hospitals, one can discern those that adopt before or during 1981 (B81) from those who adopt after 1981 (A81). Similarly for Type 4 hospitals, one can discern those that adopt before or during 1981 (B81) from those that adopt in 1982 (I82) from those that adopt after 1982 (A82).

Building the likelihood function requires that the unconditional probability of each observed adoption time be specified as a function of the conditional probabilities. ${ }^{14}$ This is done in equation 3 where the unconditional probability of each observation's adoption time is expressed as a function of $P^{1}$ and $P^{2}$. Thus, the probability that a given hospital adopted before 1981 is simply $P^{1}$, the probability of adopting before 1981. A hospital that adopted before 1983 either adopted before 1981 or during 1982 or 1983 . Thus, the contribution to the likelihood function is the unconditional probability of adopting before 1981, $P^{1}$, plus the unconditional probability of adopting during 1982 or 1983. This last term is the probability of not adopting before $1981,1-P^{1}$, times the probability of adopting in 1982 or 1983, conditional on not adopting before then, $P^{2}$. The remaining terms are derived in the same manner.

$$
\begin{aligned}
B 81 & =P^{1} \\
B 82 & =P^{1}+\left(1-P^{1}\right)\left(\alpha P^{2}\right) \\
B 83 & =P^{1}+\left(1-P^{1}\right) P^{2} \\
A 81 & =1-P^{1} \\
A 82 & =\left(1-P^{1}\right)\left(1-\alpha P^{2}\right) \\
A 83 & =\left(1-P^{1}\right)\left(1-P^{2}\right) \\
I 82 & =\left(1-P^{1}\right)\left(\alpha P^{2}\right) \\
I 83 & =\left(1-P^{1}\right)\left((1-\alpha) P^{2}\right)
\end{aligned}
$$

\footnotetext{
${ }^{14}$ Technically, the unconditional probabilities are still conditional on the sampling type. Nevertheless, they are described as unconditional for ease of exposition.
} 
Using these equations yields the conditional likelihood function in equation 4 . It is a conditional likelihood function since the expressions in equation 3 are still conditional on the sampling type. Maximizing this conditional likelihood function will provide consistent results under either of two assumptions. The stronger assumption is that the probability of a given hospital returning its survey in any given pattern (i.e. its sampling type) is independent of its adoption decisions and is, in effect, random. The weaker assumption is that the observed sampling type is a sufficient statistic for the variables governing returning the surveys. In this case, the conditional likelihood function in equation 4 will also yield consistent estimates of the parameters (Andersen 1970).

$$
\begin{aligned}
\log L= & \sum_{B 81} \ln \left(P^{1}\right)+\sum_{B 82} \ln \left(P^{1}+\left(1-P^{1}\right)\left(\alpha P^{2}\right)\right)+\sum_{B 83} \ln \left(P^{1}+\left(1-P^{1}\right) P^{2}\right)+ \\
& \sum_{A 81} \ln \left(1-P^{1}\right)+\sum_{A 82} \ln \left(\left(1-P^{1}\right)\left(1-\alpha P^{2}\right)\right)+\sum_{A 83} \ln \left(\left(1-P^{1}\right)\left(1-P^{2}\right)\right)+ \\
& \sum_{I 82} \ln \left(\left(1-P^{1}\right) \alpha P^{2}\right)+\sum_{I 83} \ln \left(\left(1-P^{1}\right)(1-\alpha) P^{2}\right)
\end{aligned}
$$

In the equations, the covariates include all of the control, regulatory and finance variables. The Medicare PPS variables only have relevance near the end of the sample. They are therefore included only in the later period, after the announcement of the move to PPS, but before its implementation.

The estimation results from this model are shown in Table 3. The first panel shows the coefficients in $P^{1}$ while the second panel shows the coefficients in $P^{2}$, including the Medicare variables. Hospital size greatly predicts adoption of all of the systems, especially in the first period. Since the model specifies probabilities, the large positive coefficients indicate that larger hospitals were more likely to adopt.

Rural hospitals are found to be consistently slower adopters of all systems, however the results are significant for only about one-half of the coefficients. For-profit and teaching hospitals exhibit this same pattern, appearing to be slower adopters of HIS overall. The one exception seems to be laboratory systems where teaching hospitals were more rapid adopters of the technology.

The Medicare PPS effects are measured by the coefficients on MB1-MB4. For the less diffused systems, there is some evidence that the impending regime shift did spur adoption. This is most 


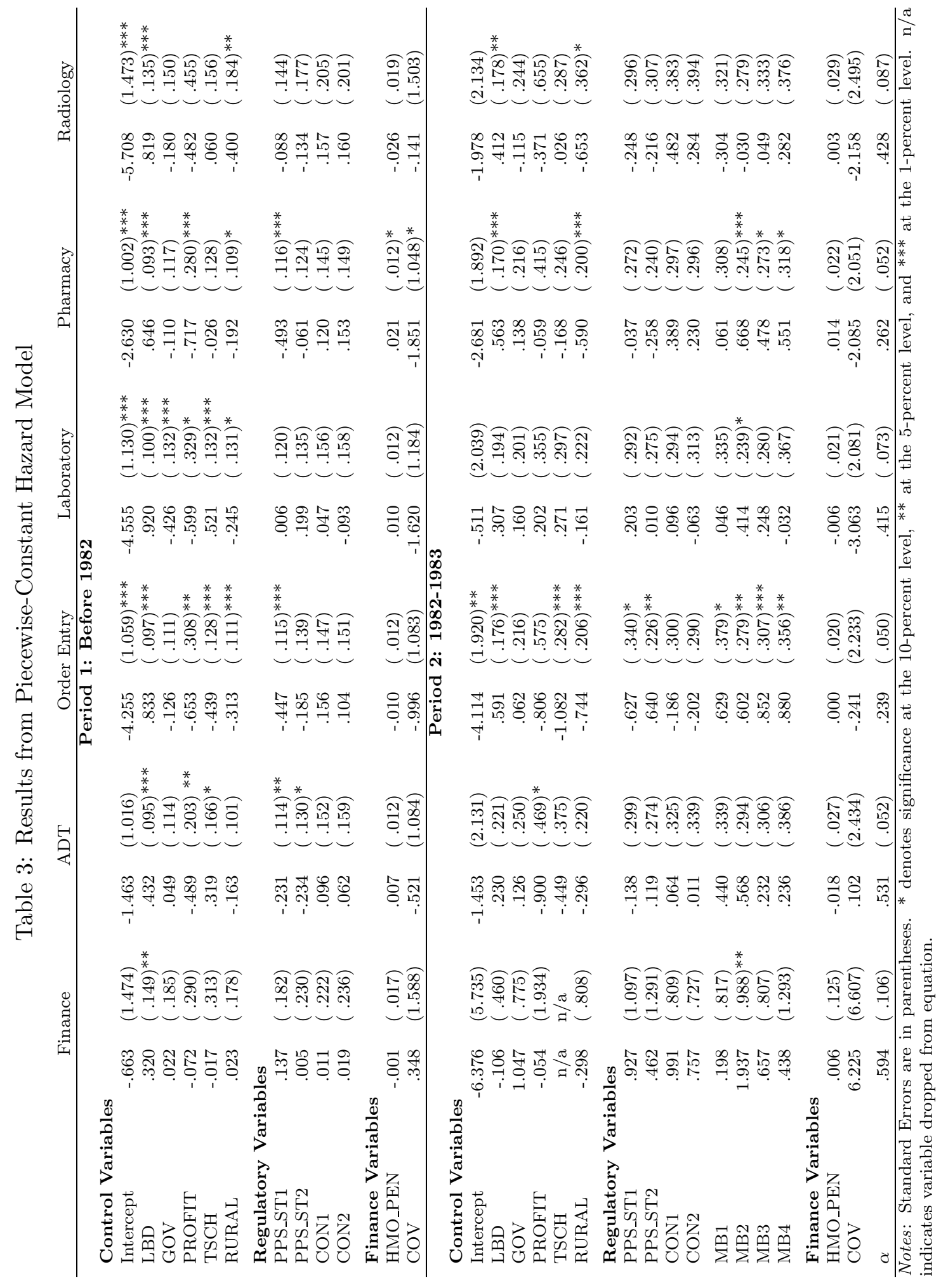


clear for order entry systems where hospitals in all groups were more likely to adopt. The results for the remaining clinical systems are less powerful, but do indicate that those facing reduced revenue were more likely to adopt in the years just before the shift.

The state regulation variables are not very precisely estimated in these estimations, since the year of adoption data is not used in this model. Still, the results show significant negative coefficients for the adoption of ADT, order entry and pharmacy systems in the early period in states with the strictest price regulation. The variables for CON regulation show no significant results, nor do the finance variables.

\subsection{Continuous Hazard Model}

The discrete model above is unable to utilize the more precise adoption times available in the data. In particular, this limits the precision of any estimates regarding the state-level PPS variables in the earlier period.

The continuous hazard model, estimated by maximum likelihood, overcomes this limitation. It uses the information contained in the adoption year in a log-logistic hazard model. The dependent variable in these regressions is the time to adoption measured from a base reference year indicating the first year any hospital had adopted the given application. ${ }^{15}$ For hospitals that adopt during the sample period, the precise value for the time to adoption is known. For hospitals that adopted before the sample period, the value is treated as left-censored; it is assumed that adoption happened on or before the given date. Hospitals that have not adopted by the last time they are seen in the data are treated as right-censored.

The covariates in this model include all of the control, regulatory and finance variables with the exception of the Medicare bite variables. The last set only have relevance near the end of the sample and are therefore excluded. The results from these regressions are shown in Table 4 .

The strongest results are among the control variables. Across all systems, larger hospitals were the fastest to adopt. This is clear from the large and statistically significant negative coefficients on

\footnotetext{
${ }^{15}$ These were derived from the current data and other sources detailing the history of HIS (e.g. Collen (1995)). For finance systems and ADT systems, the first adoption was approximately 1960. Laboratory systems were the next to be adopted in 1964, followed by order entry systems (1967), pharmacy systems (1968) and radiology systems (1970).
} 


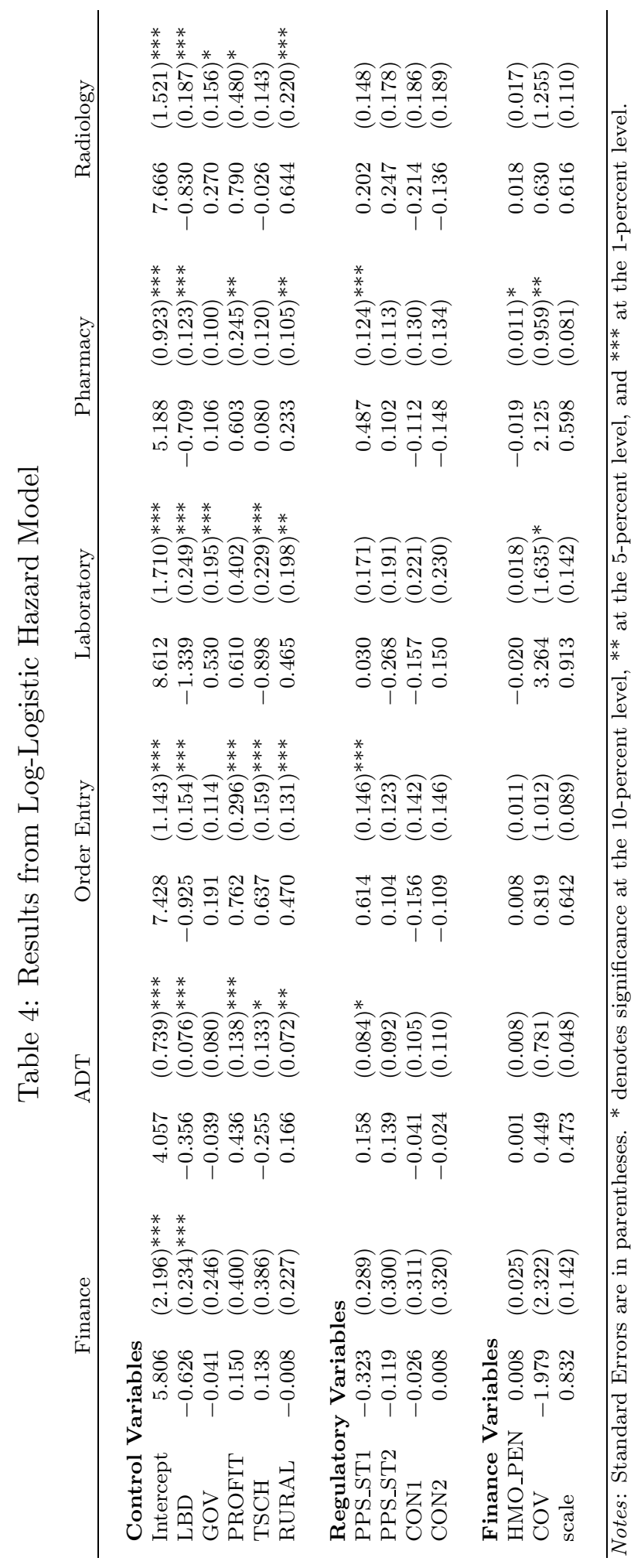


$L B D$. Since the dependent variable in this model is time to adoption, the negative sign indicates faster diffusion among the large hospitals. For-profit hospitals and those in rural areas appear to be slower adopters while teaching hospitals show mixed results. This last group appears to be slower to adopt order entry systems but faster to adopt some of the others.

The results regarding the regulatory and finance variables show some correlation with adoption of these systems. The adoption of ADT and order entry systems was delayed at hospitals in states with the most severe price regulation and hospitals in states with limited insurance coverage were slower to adopt some of the clinical systems. Interestingly, those hospitals in states with higher HMO penetration were more rapid adopters of pharmacy systems.

\section{Discussion}

The results of the estimations for order entry and pharmacy systems show the most significant results, followed by those for laboratory and ADT systems. Order entry systems require implementations throughout the hospital and thus their costs, and potential benefits, are higher and more statistically visible than the more specific clinical systems. These systems were also in the 'middle' period of diffusion. In contrast, finance systems and those used in the radiology department have very widespread and very limited diffusion respectively, offering little variation for the empirical estimations to explain.

To summarize the results more clearly, Table 5 shows the conditional probabilities, $P^{1}$ and $P^{2}$, of adoption for selected types of hospitals, based on the discrete hazard model. In each time period, the probability of adoption for a 'base' hospital is shown together with the results for other types of hospitals. The base hospital is a not-for-profit non-government hospital of median size for the universe of AHA hospitals over 100 beds (218 beds). It is located in a state with mean levels of HMO penetration (3.6\%) and health insurance coverage (85.2\%). For the second period, it is assumed that the hospital is in the group that would see revenue rise following the implementation of Medicare's PPS. ${ }^{16}$

\footnotetext{
${ }^{16}$ Since all Medicare waiver states are also in the PPS_ST1 group, these cannot be used as the base group for comparisons that examine the impact of each variable separately.
} 
In Table 5, the remaining rows show the predicted probability of adoption for hospitals with a single variable changed from the base hospital. For example, the lines labeled PROFIT show the conditional probability of adoption in the relevant period for a for-profit hospital with all the other characteristics identical to that of the base hospital. Similarly, the lines labeled PPS_ST1 show the results if the base hospital were now located in a state with the strictest price regulations. For the size variables, we include two lines: LBD-L and LBD-H show the adoption probabilities for hospitals at the 25 th (144 beds) and 75 percentiles (343 beds) of hospital size. The asterisks denote hospitals for which the predicted probability of adoption is significantly different at the indicated level from the predicted probability at the base hospital.

The results for size and location are consistent across applications. Larger hospitals are the fastest to adopt, indicating economies of scale in the use of these technologies. Even with the widespread use of outsourcing of hospital information systems, which can mitigate some of these scale economies, larger hospitals were much more rapid adopters. As an example, the predicted probability of a smaller hospital (144 beds) adopting an order entry system before 1982 is $17 \%$. For a larger hospital (343 beds), it is 41\%, nearly three times as high. For ADT systems, these probabilities are $60 \%$ and $73 \%$, a smaller but still significant difference.

The results for rural hospitals are also strong and consistent, showing that rural hospitals were slower to adopt HIS. A rural hospital's chance of adopting a pharmacy system was only $2 \%$ during the second period compared to an $8 \%$ probability for its urban counterpart. Early in the diffusion cycle, rural hospitals may not have the information about specific technologies, complementary assets (such as skilled personnel), nor viable outsourcing options. All of these limitations would raise the average cost of these systems for these hospitals, slowing rates of adoption.

The main focus of this study is the effect of the financing regime on adoption. Consistently, HMO penetration and certificate of need regulations seem to have little association with HIS adoption. HMO penetration rates were still very small at this time, which may mitigate their effect. The fact that the costs of these systems often fell under the stated CON cutoffs and that hospitals could lease these systems to avoid fixed capital expenditures limit the associations for the CON regulation variables. 


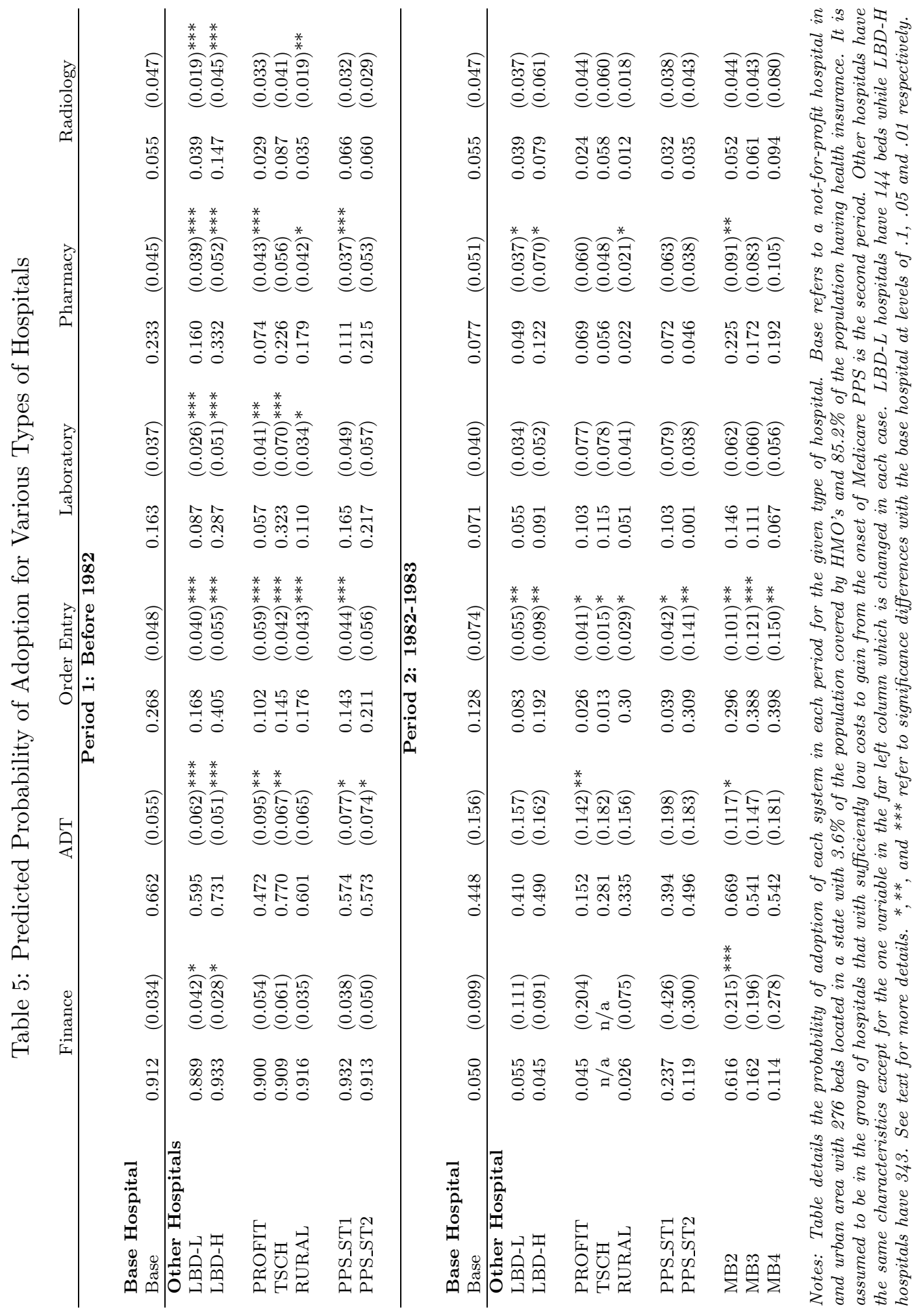


In contrast, the price regulation variables are significantly associated with HIS adoption. According to the earlier discussions, hospitals with a greater emphasis on profits should adopt slower than not-for-profit hospitals assuming a sufficient number of fee-for-service patients, and faster once the population of prospective payment patients is sufficiently high. This idea was outlined in Hypothesis 2 earlier in the paper. Since interaction terms were not included in the empirical models, this cannot be tested directly in the cross section. Still, over time (as PPS spreads), forprofit hospitals should go from being slower adopters to being faster adopters. In the early period, for-profit hospitals should lag the not-for-profits and this difference should shrink or even reverse by the second period. This is precisely the pattern in the data. Only $6 \%$ of for-profit hospitals had adopted laboratory systems before 1982 compared to $16 \%$ of not-for-profits. For pharmacy systems, only $7 \%$ of for-profit hospitals had adopted relative to $23 \%$ of not-for-profits. At the time, the majority of patients were fee-for-service; any cost savings would be passed on to the buyer of hospital services. For-profit hospitals were therefore slower to adopt: they place greater emphasis on profits and would not see any of the gains from adoption. In the second period, as the number of patients covered by PPS grows, these differences are greatly reduced.

By the second period, these gaps had narrowed. The difference between the for-profit hospitals and the base hospitals is significantly, but weakly, different for only the order entry and pharmacy systems.

The other hypothesis offered earlier was that hospitals facing greater cost pressures in the form of prospective payment are more likely to adopt cost saving technologies. The results here indicate just the opposite - hospitals in tightly price regulated states were slower adopters of HIS. For example, a hospital in one of these states had only an $11 \%$ chance of adopting a pharmacy system compared to the $23 \%$ adoption rate in a non-regulated state. Adoption of order entry systems is only $14 \%$ in the most regulated states compared to $29 \%$ at the base hospitals.

The most likely explanation for slower adoption in price regulated states is that the cost savings at this time did not warrant adoption relative to the cost of the systems. Technology in the 1970's was primarily mainframe based with mini-computer based systems beginning to appear. Given the findings about hospital size above, many hospitals could not justify HIS investments on costs 
alone. This is consistent with the evidence from the 'productivity paradox' literature which also found that computers had very limited effects during this period. ${ }^{17}$ If this is the effect driving the current results, then again the cost savings of the systems would not justify the high price tag.

Under this scenario, it is the adoption of these systems by not-for-profit hospitals that becomes the interesting question pointing to motives outside of cost savings. The leading explanations are that hospitals adopted in order to improve quality or for motives related to technological preeminence or tastes among decision-makers for new technologies. ${ }^{18}$

To assess the quality motivation, note that HIS can improve quality either by improving medical decision making among existing staff or by attracting better doctors. While the rhetoric surrounding these systems often mentioned quality of service, it is still hard to imagine great gains from these early clinical technologies. The ability of IT to attract patients and/or doctors was also likely to be muted. Unlike other highly visible technologies such as magnetic resonance imaging (MRI), the computerization of hospitals was largely invisible to patients at this time. It is hard to conceive of hospitals adopting laboratory systems to signal quality directly to patients. HIS would also have limited effect in attracting doctor's as there is substantial anecdotal evidence that many doctors resented the disruptions in work flow and procedures when new HIS were installed and would therefore not see this as a benefit.

In sum, the evidence that state regulation limited early adoption casts doubt on the ability of the earliest systems to reduce cost. The quality motivation often ascribed to hospitals is also suspect in this case, leaving technological preeminence and a taste for experimentation and learning as the most likely reasons that not-for profit hospitals adopted these clinical systems. For-profit hospitals, with more pecuniary motives, stayed away from these systems as did hospitals in more price regulated states.

Another possible explanation is that these were in fact cost saving technologies and that the decreased adoption at price-regulated states is the result of financing constraints. Under this scenario, the cost savings may have been present but yet limited access to financing for HIS hindered

\footnotetext{
${ }^{17}$ The 'productivity paradox' refers to the findings that although IT spending in the U.S. economy was high during the 1970's and 1980's, economic research could not find significant payoffs to this investment. For a summary of this research, see Brynjolfsson (1993) and Brynjolfsson and Yang (1996).

${ }^{18}$ See Teplensky et al. (1995) for a discussion of these issues in the adoption of MRI technologies.
} 
adoption in the states with greatest regulation. This idea is supported by the results in Mahajan and Schoeman (1977), where hospitals in better cash positions seem to adopt HIS faster. Of course, those results also support the idea that hospitals in good financial standing were the ones that could afford to adopt for the non-cost reasons discussed above.

By the early 1980's however, the results are quite different. Nearly $30 \%$ of hospitals facing a hit in revenue of $0 \%$ to $8 \%$ of expenses (MB2) adopted order entry systems. This compares to $17 \%$ of those for whom Medicare's change would have a positive revenue effect. Similarly, these groups had $22 \%$ and $16 \%$, respectively of adopting pharmacy systems at the same time.

To explain this result, something must have changed from the prior years when state cost control systems had a deterring effect. One set of explanations rests on differences between the state and federal payment systems. Clearly more patients were covered under the latter system yet the fundamental idea of limiting payments to fixed amounts independent of usage was the same. A more likely explanation for the change is improvement in the technology and a decrease in its cost. Either from pure technical progress or from learning, organizations' ability to use HIS to save money now justified its adoption. The ability to save money with these systems, combined with the increased incentives from PPS spurred adoption.

In conclusion, it appears that the state and federal programs did impact HIS adoption. In the early years, these systems did not have the ability to save sufficient funds to justify their expense, and adopters were motivated by factors other than cost. State price regulations are therefore associated with slower adoption. On the other hand, higher adoption rates for these systems appear to be correlated with Medicare's policy change.

To extend this research, it would be interesting to examine adoptions in the current regime where incentives to save costs are very high. In the current study, for-profit hospitals go from being slow adopters to adopting at the same rate as their not-for-profit colleagues. Is it true that today for-profit hospitals are faster to adopt cost saving innovations? Further research could also provide more precise estimations of the determinants of adoption since hospital specific variables are more readily available for later periods. In this work, only the Medicare bite variables are at the hospital level and these are rough projections. More accurate measures of a hospital's fiscal status and 
financing environment could shed light on these decisions and more broadly on the objectives of the hospital.

Lastly, it is important to note that none of the analyses above can determine if the adoption rates for these systems were optimal or to determine the net welfare effect of these regulations. Given the long learning times for these technologies and the pervasive co-invention by user firms, delayed adoption may have slowed a useful and advantageous technological development. However, if the time was not right for these technologies, then these regulations simply limited potentially wasteful spending. 


\section{References}

American Hospital Association, Annual Survey of Hospitals Various years. [Computer File].

Andersen, Erling Bernhard, "Asymptotic Properties of Conditional Maximum-likelihood Estimators," Journal of the Royal Statistical Society, Series B (Methodological), 1970, 32 (2), 283-301.

Baker, Laurence C., "Managed Care and Technology Adoption in Health Care: Evidence from Magnetic Resonance Imaging," Journal of Health Economics, 2001, 20, 395-421.

Borzekowski, Ron, "The Adoption and Cost Impact of Hospital Information Systems." PhD dissertation, Stanford University 2002.

Brynjolfsson, Erik, "The Information Paradox of Information Technology," Communications of the ACM, December 1993, $36(12), 67-77$.

and S. Yang, Information Technology and Productivity: A Review of Literature Advances in Computers

Carey, Kathleen and Theodore Stefos, "A Cost Function Approach to Measurement of Hospital Output," Health Care Financing Review, Winter 1992, 14, 115-124.

Collen, Morris Frank, A History of Medical Informatics in the United States: 1950-1990, American Medical Informatics Association, 1995.

Committee on Quality Health Care in America, Institute of Medicine, Crossing the Quality Chasm: A New Health System for the 21st Century, Washington, D.C.: National Academy Press, 2001.

Culter, David M. and Mark McClellan, "The Determinants of Technoogical Change in Heart Attack Treatment," September 1996.

Culyer, A.J. and J.P. Newhouse, eds, Handbook of Health Economics, Elsevier Science B.V., 2000. 
Dorenfest, Sheldon I., Principles, Practices, Structure, and Delivery, Gaithersburg, MD: Aspen Publishers Inc.,

_ _ "A Look Behind the Rapid Growth in Healthcare IS," Healthcare Informatics, June 1997.

Feinglass, Joe, Larry Manheim, Stephen Shortell, and Edward FX Hughes, "Documentation of 1986 State Regulatory Variables," October 1990. Northwestern University Center for Health Services and Policy Research Working Paper.

Gaumer, Gary L. et al., "Effects of State Prospective Reimbursement Programs on Hospital Mortality," Medical Care, July 1989, 27 (7), 724-736.

Globerman, Steven, "The Adoption of Computer Technology in Hospitals," The Journal of Behavioral Economics, Winter 1982, 11 (2), 67-95.

Grams, Ralph R. and Georgina Peck, "National Survey of Hospital Data Processing - 1985," Journal of Medical Systems, 1986, 10 (5/6), 423-568.

et al., "Review of Hospital Data Processing in the United States (1982-1984)," Journal of Medical Systems, 1985, 9 (4), 175-269.

Hadley, Jack, Stephen Zuckerman, and Judith Feder, "Profits and Fiscal Pressure in the Prospective Payment System: Their Imapcts on Hospitals," Inquiry, Fall 1989, 26, 354-365.

Health Care Finance Administration, U.S. Dept. of Health and Human Services, $H C F A$ Hospital Cost Reports - Minimum Dataset Various Years. [Computer File].

Herner and Company, The Use of Computers in Hospitals, National Center for Health Services Research and Development, November 1970. HSRD 70-33.

Kennedy, O. George, "Hospitals Show More Interest in Clinical Information Systems," Modern Healthcare, September 26 1986, 16 (20), 69-70.

Kimberly, John R. and Michael J. Evanisko, "Organizational Innovation: The Influence of Individual, Organizational, and Contextual Factors on Hospital Adoption of Technological 
and Administrative Innovations," Academy of Management Journal, December 1981, 24 (4), $689-713$.

Kohn, Linda T., Janet M. Corrigan, and Molla S. Donaldson, eds, To Err is Human: Building a Safer Health System, Washington, D.C.: National Academy Press, 2001.

Mahajan, Vijay and Milton E. F. Schoeman, "The Use of Computers in Hospitals: An Analysis of Adopters and Nonadopters," Interfaces, May 1977, 7 (3), 95-107.

Medical Systems Research, National Survey of Hospital Data Processing 1982-1984. [Computer File].

Moch, Michael K. and Edward V. Morse, "Size, Centralization and Organizational Adoption of Innovations," American Sociological Review, October 1977, 42, 716-725.

National Center for Health Statistics, Hyattsville, Maryland: National Center for Health Statistics, 2000.

Newhouse, Joseph P., "Toward a Theory of Nonprofit Institutions: An Economic Model of a Hospital," American Economic Review, 1970, 60 (1), 64-74.

Pauly, M.V. and M. Redisch, "The Not-for-Profit as a Physician's Cooperative," American Economic Review, 1973, 63 (1), 87-99.

Romeo, Anthony, Judith Wagner, and Robert Lee, "Prospective Reimbursement and the Diffusion of New Technologies in Hospitals," Journal of Health Economics, 1984, 3, 1-24.

Rosko, Michael D. and Robert W. Broyles, The Economics of Health Care: A Reference Handbook, New York: Greenwood Press, 1988.

Salkever, David S., "Regulation of Prices and Investement in Hospitals in the United States." In Culyer and Newhouse, eds (2000) chapter 28.

Sheldon I. Dorenfest \& Associates, Ltd., Hospital Information Systems, State of the Art, 1985 Edition 1985. 
Sloan, Frank A., "Not-for-Profit Ownership and Hospital Behavior." In Culyer and Newhouse, eds (2000) chapter 28.

Teplensky, Jill D., Mark V. Pauly, John R. Kimberly, Alan L. Hilman, and J. Sanford Schwartz, "Hospital Adoption of Medical Technology: An Empirical Test of Alternative Models," Health Services Research, 1995, 30 (3), 437-465.

U.S. Census Bureau, Current Population Survey 1980. [Computer File]. 